\title{
Wearable Sensor Application for Integrated Early Warning and Health Surveillance
}

\author{
Lauren E. Charles*, Devin P. Wright, Zhuanyi Huang, Cree White, FNU Anubhav, \\ Yuanqing Jin and Michael Henry
}

Pacific Northwest National Laboratory, Richland, WA, USA

\section{Objective}

The Wearable Sensor Application developed by Pacific Northwest National Laboratory (PNNL) provides an early warning system for stressors to individual and group health using physiologic and environmental indicators. The application integrates health monitoring parameters from wearable sensors, e.g., temperature and heart rate, with relevant environmental parameters, e.g., weather and landscape data, and calculates the corresponding physiological strain index. The information is presented to the analyst in a group and individual view with real-time alerting of abnormal health parameters. This application is the first of its kind being developed for integration into the Defense Threat Reduction Agency's Biosurveillance Ecosystem (BSVE).

\section{Introduction}

Wearable devices are a low cost, minimally invasive way to monitor health. Sensor data provides real-time physiological indictors of an individual's health status without the requirement of health care professionals or facilities. Information gleamed from wearable sensors can be used to better understand physiological stressors and prodromal symptoms. In addition, this data can be used to monitor individuals that are in high risk of health-related problems.

However, raw data from wearable sensors can be overwhelming to process and laborious to monitor for an individual and, even more so, for a group of individuals. Often specific combination of ranges of sensor readings are indicative of changes to health status and need to be evaluated together or used to calculate specific signal parameters. In addition, the environment surrounding the individual needs to be considered when interpreting the data. To address these issues, PNNL has developed an application that collects, analyzes, and integrates wearable sensor data with geographic landscape and weather information to provide a real-time early alert and situational awareness tool for monitoring the health of groups and individuals.

\section{Methods}

The prototype application described here was a product of PNNL's BSVE Application Development Competition. The final product that will be deployed in the BSVE is currently under development by PNNL and will vary slightly in the exact design and architecture described.

Data. Wearable sensor data was collected from the Rim2Rim (R2R) Watch Study of individuals hiking the Grand Canyon in Arizona [1]. Weather information was obtained from nearby weather stations and mapping features were derived from Google Maps.

Calculations. A physiological Strain Index (PSI) was calculated using core temperature estimates derived through a Kalman Filter approach and heart rate [2,3].

Application. The prototype backend application development was based in Python with a MongoDB. The front-end development was built using a scalable architecture and modular approach with components in React and D3.

\section{Results}

A prototype application was developed this past summer through the PNNL BSVE App Competition (Fig 1). The application was aimed at visualizing wearable sensor data from the Grand Canyon R2R hike dataset. Simulated real-time analysis was used to calculate health status of individuals hiking based on measured physiological parameters and to alert to individuals with signs of physiologic health stress. Visualization tools were incorporated to enable sensor data for individuals and the group to be viewed simultaneously along with pertinent weather, geographic, and elevation data.

Many features described in the prototype application will be incorporated into the final BSVE application. The key changes will be 1) the ability to select given time periods for viewing historical data as well as the real-time data collection, 2) environmental data and map view will come from BSVE internal data sources, and 3) the alerts will provide more information and have their own page for reviewing.

\section{Conclusions}

The Wearable Sensor Application developed by PNNL for integration into the BSVE provides an early warning system for individual and group health using physiologic and environmental parameters. The application highlights health status from wearable sensors and relevant environmental parameters while monitoring a calculated physiological strain index. With this tool, an analyst can easily monitor the health of individuals and groups with the aid of realtime alerting tool for early detection of abnormal health parameters.

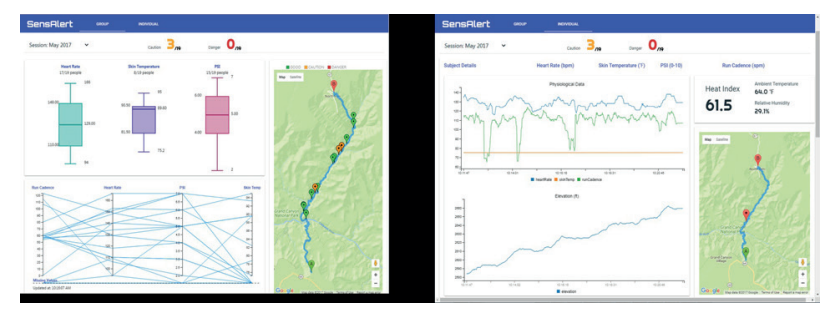

Figure 1. Wearable sensor application prototype group page (left) and individual page (right). Group views include box \& whiskers and parallel coordinate plots of sensor readings, map view of individuals with alert status, and alert counter. Individual views include alerts, time series of sensors, weather, map view, and elevation overtime.

\section{Keywords}

Wearable Sensors; Health Surveillance; Early Warning; Real-time Data; Physiologic Strain Index

\section{Acknowledgments}

This work was funded by the Defense Threat Reduction Agency (project number CB10190). 


\section{ISDS 2018 Conference Abstracts}

\section{References}

[1] Aviña GE, Abbott R, Anderson-Bergman C, et al. 2017. Rim-to-Rim Wearables At the Canyon for Health (R2R WATCH): Experimental Design and Methodology. HCI International 2017 Conference Proceedings.

[2] Buller MJ, Tharion WT, Cheuvront SN, et al. 2013. Estimation of Human Core Temperature from Sequential Heart Rate Observations. Institude of Physics and Engineering in Medicine - Physiological Measurement 34(7)

[3] Moran DS, Shitzer A, \& Pandolf KB. 1998. A Physiological Strain Index to Evaluate Heat Stress. American Journal of Physiology Regulatory, Integrative and Comparative Physiology 275(1): R129-R134.

\section{*Lauren E. Charles}

E-mail: lauren.charles@pnnl.gov 